\title{
Effect of Weight Loss on Symptoms of Polycystic Ovarian Syndrome among Women of Reproductive Age
}

\author{
Anita Dileep ${ }^{\mathrm{a}}$ Manal Ahmed Faek Samy ${ }^{\mathrm{a}}$ Nazneen Hussain ${ }^{\mathrm{b}}$ \\ Sham Zain Alabdind ${ }^{\mathrm{C}}$ \\ aWomen Health Unit Department, PHCS, AI Mizhar Health Centre, Dubai Health Authority, Dubai, UAE; \\ ${ }^{\mathrm{b}}$ Family Medicine Department, PHCS, AI Khawaneej Health Centre, Dubai Health Authority, Dubai, UAE; \\ 'Clinical Pharmacy Department, Ajman University-College of Pharmacy and Health Sciences, Ajman, UAE
}

\section{Keywords}

Polycystic ovarian syndrome - Weight loss · Obesity · Insulin resistance $\cdot$ Metformin

\begin{abstract}
Background: The polycystic ovarian syndrome (PCOS) is a common endocrine disease, which affects women of childbearing age. Its exact etiology is not yet fully understood, but there have been a number of theories and hypotheses which suggested that it is related to multiple factors. However, the most common cause is the insulin resistance, which contributed in development of PCOS and weight gain as well. Aim: This study aims to assess the impact of weight control on the management of PCOS among obese patients. Methods: This is a retrospective observational study conducted on 68 obese PCOS women at different primary healthcare centers in Dubai. Patients were included only if they were following a healthy lifestyle, using metformin $(750-1,000 \mathrm{mg})$ for at least 3-9 months, and lost at least $5.0-10.0 \%$ of their baseline body weights. The follow-up period was for 1 year based on their medical records. Data were analyzed and represented as mean \pm standard deviation of continues measures, while the $x^{2}$ test, independent Student's $t$ test, and one-way ANOVA test were performed for comparing numerical variables
\end{abstract}

between different groups of categorical variables. $p$ value was considered significant at $<0.05$. Results: Mean age of the patients was $26.1 \pm 6.5$ years, and the average baseline BMI was $34.7 \pm 4.3 \mathrm{~kg} / \mathrm{m}^{2}$. The most frequently reported clinical feature of PCOS was irregular menstruation ( $n=38,55.9 \%)$. Majority of the patients (80.0\%) who had a successful weight loss (median of $8.0[3,10] \mathrm{kg}$ ) during the 1-year follow-up reported a significant clinical improvement, while those reported no clinical improvement did not actually have a considerable weight loss (median of $4[2,5] \mathrm{kg})(p<0.001)$. Additionally, the duration of treatment of 9 months was significantly respectable for achieving higher weight loss than less durations $(p=0.002)$. Furthermore, the only factor which contributed to clinical improvement of PCOS was the baseline BMI $(p<0.001)$. Conclusion: This study demonstrated that following an appropriate care plan by a combination of lifestyle modifications and metformin was effective in achieving a successful weight loss, which resulted in a significant improvement in the clinical features of the PCOS. Further studies are recommended to raise awareness of women regarding the PCOS and to encourage them to communicate with healthcare providers for initiating their selfmanagement care.

(c) 2021 The Author(s). Published by S. Karger AG, Basel karger@karger.com

www.karger.com/dmj

(C) 2021 The Author(s).

Published by S. Karger AG, Basel
Correspondence to:

Anita Dileep, ADileep@ dha.gov.ae

Karger ${ }^{\prime}=$ 


\section{Introduction}

The polycystic ovarian syndrome (PCOS) is a common endocrine disease, which affects women of childbearing age. This syndrome has been discovered by Stein and Leventhal in 1935 and is also called as "Stein-Leventhal syndrome." The incidence rate of PCOS ranges between 5.0 and $13.0 \%$ depending on many factors such as ethnicity, region, and hormonal disturbance [1]. According to the Rotterdam diagnostic criteria, prevalence of PCOS varies between 3.0 and $26.0 \%$ [2,3]. A systematic review and meta-analysis published on this topic in 2017 reported that this huge prevalence might be related to hereditary and ethnicity as well as certain lifestyle-related influences. As far as Middle East/GCC are concerned, many studies have been published reporting high prevalence of PCOS in the range of $20.0-25.0 \%$ in these regions [4].

Despite the availability of widespread research on this topic, its exact etiology is not yet fully understood. There have been a number of theories and hypotheses suggested on this topic, and the conclusion of most research is that it is not a single-factor disease but rather multiple factors play a role together including genetic factors as shown in previous studies $[5,6]$.

Generally, the PCOS women present the following clinical symptoms: anovulatory menstrual irregularities, acne hirsutism, male pattern baldness, acanthosis nigricans, sleep apnea, infertility, and obesity with high distribution of central fat [7]. Obesity is the most common feature of PCOS and prevalent among $>50.0 \%$ of PCOS women. Most of the research studies associated the presence of obesity among PCOS patients due to high insulin levels, which result from insulin resistance as an important factor of adipogenesis, lipogenesis, and lipolysis inhibition [8]. However, it is still debatable whether PCOS leads to obesity or vice versa [9]. Moreover, childhood obesity is a well-documented risk factor for PCOS, as obese girls are at higher risk of insulin resistance with subsequent hyperinsulinemia and its consequences including metabolic syndrome and PCOS later in life [10]. Therefore, obesity plays an important role in the clinical and biochemical presentation of PCOS; hence, weight loss in the range of $5.0-10.0 \%$ should be considered as a first-line treatment in the management of this common endocrine pathology. Life style modifications include healthy balanced diet such as having regular meals especially breakfast, diet rich in fruit, vegetables, and whole food (whole meal bread, whole grain cereals, brown rice, and whole meal pasta), lean meat, fish, and chicken while low in simple carbohydrates (sugar). In addition to diet, regular exercise for at least $30 \mathrm{~min}$ per day 3 times/week is an important element of the weight control program [11]. Thus, this retrospective study aims to evaluate the impact of weight control measures on the clinical outcomes of PCOS women over a period of 1-year follow-up.

\section{Methodology}

\section{Design}

This is a longitudinal retrospective observational study that was carried out on females suffering from PCOS. Data were obtained from patients' records retrospectively at different primary healthcare centers in Dubai from January 2017 to December 2018, and each patient was followed up at 12 months for assessment of clinical improvement and consultation in the clinics. The study was approved by the Dubai Scientific Research Ethics Committee "DSREC-08/2019_10."

\section{Participants}

This study recruited initially 239 premenopausal cases with PCOS, but only 68 patients of them were included in this study according to the inclusion criteria. The selected patients should be either overweight or obese with a BMI of $\geq 24 \mathrm{~kg} / \mathrm{m}^{2}$ and lost at least $5.0-10.0 \%$ of their baseline weight. In addition, patients should be on diet and use metformin (Glucophage) 750-1,000 mg daily for 3-9 months and then followed up at 12 months. The study excluded thin lean $(\mathrm{BMI} \leq 18)$ women with PCOS or women with PCOSrelated infertility.

\section{Variables and Procedures}

Diagnosed cases of PCOS were recruited electronically by using ICD Coding for disease. The studied variables in this study included age, age of menarche, marital status, ethnicity, family history, clinical features of PCOS, and BMI at baseline and percentage of weight loss. The clinical improvement was evaluated at 1-year follow-up.

\section{Statistical Analysis}

Data analysis was performed using the SPSS software (Version 24.0). Descriptive data were represented as mean \pm standard deviation of continues measures (age and $\mathrm{BMI}$ ), while categorical data were represented as frequencies and percentages. Categorical variables were compared with the $\chi^{2}$ test if assumptions fulfilled; otherwise, the Fisher exact test was used. Independent Student's $t$ test and one-way ANOVA test were applied to compare continuous variables if normality assumption met. For nonnormally distributed data, medians, Mann-Whitney U test, and Kruskal-Wallis test were applied. $p$ value $<0.05$ was considered significant.

\section{Results}

\section{Baseline Patient Characteristics}

Table 1 represents the baseline characteristics of the patients. A total of 68 cases met the criteria and were reviewed in this study. Mean age of the patients was $26.1 \pm$ 6.5 , and the average age of menarche was $11.9 \pm 1.1$ years. 


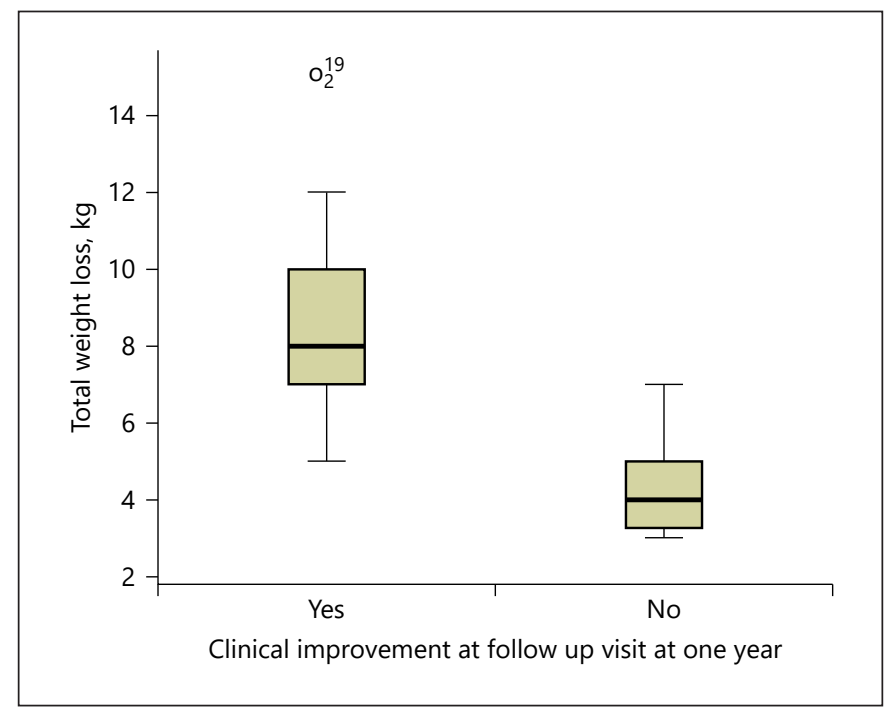

Fig. 1. Total weight loss among patients who reported or did not report a clinical improvement.

Majority of them were single $(n=46,67.6 \%)$ and were from the middle east of Asia ( $n=53,77.9 \%)$. Most of the patients had a family history of PCOS $(n=39,57.4 \%)$. At baseline, menstrual irregularity was present in more than half of the sample $(n=38,55.9 \%)$. The mean baseline BMI was at the higher side $34.7 \pm 4.3 \mathrm{~kg} / \mathrm{m}^{2}$.

\section{Clinical Outcomes after Successful Weight Loss}

The average duration of drug treatment in the group was $7.1 \pm 1.7$ months, which resulted in a mean weight loss of $7.5 \pm 2.7 \mathrm{~kg}$. Clinical improvement was self-reported by almost $80 \%(n=53)$ of the patients at the end of 12-month follow-up. Weight loss between the groups who reported clinical improvement compared to those who did not was not normally distributed. Median weight loss among the group who reported a clinical improvement after drug therapy was $8.0[3,10] \mathrm{kg}$ compared to the median weight loss of $4[2,5] \mathrm{kg}$ among the group with no clinical improvement. The difference in weight loss between both groups was statistically significant $(p<$ 0.001) as shown in Figure 1.

The duration of drug therapy was divided into 3 groups: 3,6 , and 9 months. More than half of the patients $(n=36,52.9 \%)$ received the treatment for 6 months, and $42.6 \%(n=29)$ received it for 9 months. Mean weight loss among those who completed their treatment for 9 months $(8.9 \pm 2.8 \mathrm{~kg})$ was significantly high $(p=0.002)$ compared to other durations $(5.6 \pm 2.9$ at 3 months and $6.7 \pm 2.3$ at 6 months) as shown in Figure 2.

Effect of Weight Loss on Women with Polycystic Ovarian Syndrome

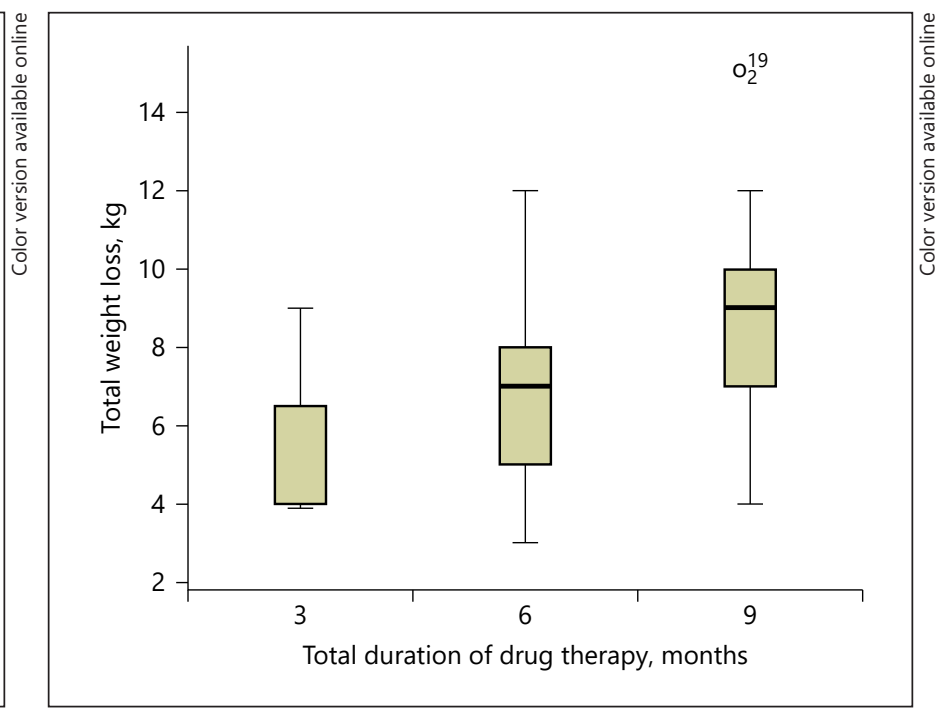

Fig. 2. Total weight loss at different durations of treatment.

Table 1. Baseline patient characteristics $(N=68)$

$\begin{array}{ll}\text { Marital status } & \\ \quad \text { Single } & 46(67.6) \\ \quad \text { Married } & 22(32.4) \\ \text { Ethnicity } & \\ \quad \text { Middle East Asian } & 53(77.9) \\ \quad \text { South East Asian } & 15(22.1) \\ \text { Family history of PCOS } & \\ \quad \text { Yes } & 39(57.4) \\ \quad \text { No } & 29(42.6) \\ \text { Presenting complaint } & \\ \quad \text { Menstrual irregularity } & 38(55.9) \\ \quad \text { Hirsutism/acne } & - \\ \quad \text { Both } & 30(44.1) \\ \text { Age, years } & 26.1 \pm 6.5 \\ \text { Age at menarche, years } & 11.9 \pm 1.1 \\ \text { Baseline BMI, kg/m }{ }^{2} & 34.7 \pm 4.3\end{array}$

Values denote $n(\%)$ or mean \pm SD. PCOS, polycystic ovarian syndrome.

\section{Predictors of Clinical Improvement after Successful Weight Loss}

Clinical improvement of PCOS after the treatment was not associated with any of the baseline characteristics. The only factor that affected the clinical improvement significantly was the baseline BMI $(p<0.001)$, as those 
Table 2. Predictors of clinical improvement after successful weight loss

\begin{tabular}{|c|c|c|c|c|}
\hline \multicolumn{5}{|l|}{ Marital status } \\
\hline Single & $46(67.6)$ & $36(67.9)$ & $10(66.6)$ & \multirow[t]{2}{*}{0.93} \\
\hline Married & $22(32.4)$ & $17(32.1)$ & $5(33.3)$ & \\
\hline \multicolumn{5}{|l|}{ Ethnicity } \\
\hline \multicolumn{5}{|l|}{ Family history of PCOS } \\
\hline Yes & $39(57.4)$ & $32(60.4)$ & $7(46.7)$ & \multirow[t]{2}{*}{0.34} \\
\hline No & $29(42.6)$ & $21(39.6)$ & $8(53.3)$ & \\
\hline \multicolumn{5}{|l|}{ Presenting complaint } \\
\hline Menstrual irregularity & $38(55.9)$ & $27(51.0)$ & $11(73.3)$ & \multirow[t]{2}{*}{0.12} \\
\hline Hirsutism/acne & - & - & - & \\
\hline 9 months & $29(42.6)$ & $25(47.1)$ & $4(26.6)$ & 0.09 \\
\hline Age, years & $26.1 \pm 6.4$ & $25.9 \pm 6.3$ & $26.6 \pm 7.1$ & 0.72 \\
\hline Age at menarche, years & $11.8 \pm 1.1$ & $11.9 \pm 1.1$ & $11.7 \pm 1.0$ & 0.63 \\
\hline Body mass index, $\mathrm{kg} / \mathrm{m}^{2}$ & $34.6 \pm 4.2$ & $33.7 \pm 3.7$ & $37.6 \pm 4.5$ & $0.001^{*}$ \\
\hline
\end{tabular}

Values denote $n(\%)$ or mean \pm SD. ${ }^{*}$ Significance at $p$ value $<0.05$. PCOS, polycystic ovarian syndrome.

with lower BMI values reported better clinical outcomes after the treatment compared to those with high BMI values (Table 2).

\section{Discussion}

This study discusses the impact of weight control on PCOS symptoms among obese women at reproductive age. Patients were supposed to follow a healthy diet and take metformin for at least 6-9 months and were screened for clinical improvement if they have lost $\geq 5.0 \%$ of their body weight within 1-year follow-up.

According to our data, obesity was prevalent among all of our PCOS patients (mean BMI: $34.7 \pm 4.3 \mathrm{~kg} / \mathrm{m}^{2}$ ). This is consistent with the literature, where some studies reported prevalence of obesity among at least $50.0-70.0 \%$ of PCOS patients $[12,13]$. The prevalence of obesity among PCOS patients has remained unclear; however, most researchers have attributed that to either hyperinsulinemia caused by insulin resistance or hyperandrogenism $[8,12]$. Obesity is not necessarily to be present among PCOS patients, but the insulin resistance and develop- ment of hyperinsulinemia along with the hormonal disturbance precipitate the clinical features of PCOS [12$14]$.

Based on our findings, clinical improvement of PCOS symptoms was reported by $80.0 \%$ of our patients, who had a successful weight loss. Likewise, several reports addressed the full or at least partial absence of the symptoms and PCOS phenotype after weight loss $[15,16]$. Hence, it is important to take into account the consequent value of weight loss for improving PCOS symptoms. Modifications in lifestyles exist again as the first-phase care plan for patients with PCOS.

This study revealed that a 9-month metformin therapy along with lifestyle modification was significantly effective in reducing body weight and improving the clinical features of PCOS $(p=0.002)$. This is similar to a previous study, which showed lower levels of insulin achieved after $\geq 5.0 \%$ loss in body weight, thus improving the PCOS clinical symptoms [17]. Accordingly, weight loss after using metformin is related to a reduced appetite associated with metformin regular use [18]. Studies have shown that metformin use among lean PCOS patients is equally effective among the obese ones [19]. However, there are 
some conflicting studies if metformin affects the ovarian steroid-genesis [20, 21].

Although women with PCOS had higher adiposity, to some extent [22], the great participation weight control is dependent on BMI adjustment and other demographic factors. It means that the status of PCOS itself is correlated with a higher incidence of weight control practice. This can be justified by the interest of PCOS women to consider themselves at high risk of obesity [23], as well as diagnosis of overweight and obesity among patients with associated comorbidities [24]. These results are consistent with the question of weight loss being the most common health issue among women with PCOS [25]. Additionally, a previous study reported that massive weight loss among morbidly obese patients resulted in a complete resolution of PCOS [26].

In the current study, clinical improvement in PCOS after the treatment was not associated with any of the sociodemographics. However, it was significantly associated with reduced BMI over the follow-up period ( $p<$ 0.001 ). Likewise, data from the literature stated that clinical features of PCOS (at all ages) are significantly determined by BMI levels [27]. Further assessment of the impact of sociodemographics on improving the clinical symptoms of PCOS should be studied, in order to implement suitable strategies in the management plan and raise awareness of women to either prevent the occurrence of PCOS or follow evidence-based care plan to achieve the best outcomes.

The studies found also had moderate to high bias risk and were generally small. Most of them did not explicitly rule out the status of PCOS in their systems. To enhance the consistency of the analysis, future studies should tackle these methodological shortcomings. Also, to enhance the reliability and feasibility of future studies, thorough documentation of inclusion and exclusion requirements, increased sample numbers, retention levels, anthropometric assessment protocols, and study publishing protocols are suggested. Moreover, endpoint monitoring may also be improved across the spectrum of health effects associated with obesity in PCOS and by using a prespeci- fied list of results similar to those recommended for infertility care.

In conclusion, this study revealed that following an appropriate care plan by a combination of lifestyle modifications and metformin was effective in achieving a successful weight loss, which resulted in a significant improvement in the clinical features of the PCOS. Further studies are recommended to raise awareness of women regarding the PCOS through educational materials and workshops as well as internet-based publications. In addition, women should be encouraged to communicate with the healthcare providers for initiating their selfmanagement care.

\section{Acknowledgement}

The study authors would like to thank the DHA Women Health Unit-Primary Healthcare Centers for their cooperation and facilitation of conducting this study.

\section{Statement of Ethics}

The study was approved by the Dubai Scientific Research Ethics Committee "DSREC-08/2019_10."

\section{Conflict of Interest Statement}

All authors have no conflicts of interest to declare.

\section{Funding Sources}

The authors did not receive any funding.

\section{Author Contributions}

Anita Dileep: writing the proposal and getting the ethical approval, drafting the manuscript, and revising the final manuscript. Manal Ahmed Faek Samy: collecting data. Nazneen Hussain: collecting data. Sham Zain Alabdin: analyzing and interpreting the data.

\section{References}

1 Sam S, Dunaif A. Polycystic ovary syndrome: syndrome XX? Trends Endocrinol Metab. 2003 Oct;14(8):365-70.

2 Hashemipour M, Amini M, Iranpour R, Sadri GH, Javaheri N, Haghighi S, et al. Prevalence of congenital hypothyroidism in Isfahan, Iran: results of a survey on 20,000 neonates. Horm Res. 2004;62(2):79-83.
3 Driscoll DA. Polycystic ovary syndrome in adolescence. Ann N Y Acad Sci. 2003;997(1): 49-55.

4 Ding T, Hardiman PJ, Petersen I, Wang FF, $\mathrm{Qu} \mathrm{F}$, Baio G. The prevalence of polycystic ovary syndrome in reproductive-aged women of different ethnicity: a systematic review and meta-analysis. Oncotarget. 2017 Nov 10; 8(56):96351.
Effect of Weight Loss on Women with Polycystic Ovarian Syndrome
Dubai Med J 2021;4:127-132 DOI: $10.1159 / 000514025$ 
5 Escobar-Morreale HF, Luque-Ramírez M, San Millán JL. The molecular-genetic basis of functional hyperandrogenism and the polycystic ovary syndrome. Endocr Rev. 2005 Apr;26(2):251-82.

6 Yildiz BO, Goodarzi MO, Guo X, Rotter JI, Azziz R. Heritability of dehydroepiandrosterone sulfate in women with polycystic ovary syndrome and their sisters. Fertil Steril. 2006 Dec;86(6):1688-93.

7 MayoClinic. Polycystic ovary syndrome (PCOS) - symptoms and causes - Mayo Clinic [Internet]. Polycystic Ovary Syndr PCOS. 2017 [cited 2020 Aug 30]. Available from: https: //www.mayoclinic.org/diseases-conditions/pcos/symptoms-causes/syc-20353439.

8 Corbould A, Dunaif A. The adipose cell lineage is not intrinsically insulin resistant in polycystic ovary syndrome. Metab Clin Exp. 2007 May;56(5):716-22.

9 Kamangar F, Okhovat JP, Schmidt T, Beshay A, Pasch L, Cedars MI, et al. Polycystic ovary syndrome: special diagnostic and therapeutic considerations for children. Pediatr Dermatol. 2015;32(5):571-8.

10 Pasquali R, Stener-Victorin E, Yildiz BO, Duleba AJ, Hoeger K, Mason H, et al. PCOS forum: research in polycystic ovary syndrome today and tomorrow. Clin Endocrinol. 2011; 74(4):424-33.

11 Hosseini MS, Dizavi A, Rostami H, Parastouei K, Esfandiari S. Healthy eating index in women with polycystic ovary syndrome: a case-control study. Int J Reprod Biomed. 2017 Sep;15(9):575.

12 Baptiste CG, Battista MC, Trottier A, Baillargeon JP. Insulin and hyperandrogenism in women with polycystic ovary syndrome. J Steroid Biochem Mol Biol. 2010 Oct;122(13):42-52.
13 Baillargeon J-P, Nestler JE. Polycystic ovary syndrome: a syndrome of ovarian hypersensitivity to insulin? J Clin Endocrinol Metab. 2006;91(1):22-4.

14 Gambineri A, Pelusi C, Vicennati V, Pagotto U, Pasquali R. Obesity and the polycystic ovary syndrome. Int J Obes Relat Metab Disord. 2002;26(7):883-96.

15 Barber TM, Hanson P, Weickert MO, Franks S. Obesity and polycystic ovary syndrome: implications for pathogenesis and novel management strategies. Clin Med Insights Reprod Health. 2019 Sep;13.

16 Pasquali R, Gambineri A, Cavazza C, Ibarra Gasparini D, Ciampaglia W, Cognigni GE, et al. Heterogeneity in the responsiveness to long-term lifestyle intervention and predictability in obese women with polycystic ovary syndrome. Eur J Endocrinol. 2011;164(1):5360.

17 Moran L, Norman RJ. Understanding and managing disturbances in insulin metabolism and body weight in women with polycystic ovary syndrome. Best Pract Res Clin Obstet Gynaecol. 2004 Oct;18(5):719-36.

18 Glueck CJ, Aregawi D, Agloria M, Winiarska M, Sieve L, Wang P. Sustainability of $8 \%$ weight loss, reduction of insulin resistance, and amelioration of atherogenic-metabolic risk factors over 4 years by metformin-diet in women with polycystic ovary syndrome. Metab Clin Exp. 2006;55(12):1582-9.

19 Maciel GA, Soares Júnior JM, Alves da Motta EL, Abi Haidar M, de Lima GR, Baracat EC. Nonobese women with polycystic ovary syndrome respond better than obese women to treatment with metformin. Fertil Steril. 2004; 81(2):355-60.

20 Arlt W, Auchus RJ, Miller WL. Thiazolidinediones but not metformin directly inhibit the steroidogenic enzymes P450c17 and 3betahydroxysteroid dehydrogenase. J Biol Chem. 2001;276(20):16767-71.
21 Mansfield R, Galea R, Brincat M, Hole D, Mason $\mathrm{H}$. Metformin has direct effects on human ovarian steroidogenesis. Fertil Steril. 2003; 79(4):956-62.

22 Moran LJ, Ko H, Misso M, Marsh K, Noakes $\mathrm{M}$, Talbot $\mathrm{M}$, et al. Dietary composition in the treatment of polycystic ovary syndrome: a systematic review to inform evidence-based guidelines. J Acad Nutr Diet. 2013;113(4): $520-45$.

23 Moran L, Gibson-Helm M, Teede H, Deeks A. Polycystic ovary syndrome: a biopsychosocial understanding in young women to improve knowledge and treatment options. J Psychosom Obstet Gynaecol. 2010 Mar;31(1):24-31.

24 Ossolinski G, Jiwa M, McManus A. Weight management practices and evidence for weight loss through primary care: a brief review. Curr Med Res Opin. 2015;31(11):201120.

25 Gibson-Helm M, Teede H, Dunaif A, Dokras A. Delayed diagnosis and a lack of information associated with dissatisfaction in women with polycystic ovary syndrome. J Clin Endocrinol Metab. 2017;102(2):604-12.

26 Escobar-Morreale HF, Botella-Carretero JI, Alvarez-Blasco F, Sancho J, San Millán JL. The polycystic ovary syndrome associated with morbid obesity may resolve after weight loss induced by bariatric surgery. J Clin Endocrinol Metab. 2005;90(12):6364-9.

27 Ollila M-ME, Piltonen T, Puukka K, Ruokonen A, Järvelin M-R, Tapanainen JS, et al. Weight gain and dyslipidemia in early adulthood associate with polycystic ovary syndrome: prospective cohort study. J Clin Endocrinol Metab. 2016;101(2):739-47. 